EPJ Web of Conferences 19, 01003 (2012)

DOI: $10.1051 /$ epjconf/20121901003

(C) Owned by the authors, published by EDP Sciences, 2012

\title{
Andromeda and the Milky Way: Twin sisters, distant relations, or strangers in the night?
}

\author{
A. McConnachie ${ }^{1, a}$, J. Richardson ${ }^{2}$, D. Mackey ${ }^{3}$ and the PAndAS collaboration \\ ${ }^{1}$ NRC Herzberg Institute of Astrophysics, Victoria, British Columbia, Canada, V9E 2E7 \\ ${ }^{2}$ Institute of Astronomy, University of Cambridge, Madingley Road, Cambridge CB3 OHA, UK \\ ${ }^{3}$ Research School of Astronomy \& Astrophysics, Australian National University, Mt. Stromlo \\ Observatory, Weston Creek, ACT 2611, Australia
}

\begin{abstract}
I summarize some recent key results from the Pan-Andromeda Archaeological Survey (PAndAS), in particular showing how recent discoveries in and around M31 compare to the known structure of the Milky Way and its satellite population.
\end{abstract}

\section{INTRODUCTION}

The Pan-Andromeda Archaeological Survey (PAndAS) is a Large Program on the Canada-FranceHawaii telescope that ran from S08B-S10B. It obtained over 400 square degrees of contiguous MegaCam imaging around M31 and M33, reaching out to maximum projected radii of $150 \mathrm{kpc}$ from $\mathrm{M} 31$ and $50 \mathrm{kpc}$ from M33, in the $\mathrm{g}$ and $\mathrm{i}$ bands. These observations are deep enough to resolve the $>4$ magnitudes down the red giant branch of M31 (nearly to the horizontal branch level; see [9] for more details).

\section{ASSEMBLING THE PUZZLE OF...}

Figure 1 is a reproduction of Figure 1 from [11]. Here, we show the spatial density distribution of candidate metal-poor RGB stars in the environs of M31 and M33, selected using a color-cut in colormagnitude space.

\section{1 ...the Andromeda sub-group}

Figure 1 shows a vast array of substructure in the area surrounding M31 and M33, down to equivalent surface brightnesses of 32-33 mags $\operatorname{arcsec}^{-2}$. Several of the streams visible are $>50 \mathrm{kpc}$ in extent, and the stars extend to vast radii (to beyond the edge of the survey). For comparison to the MW, the SDSS currently maps the MW halo out to radii of around $40 \mathrm{kpc}$ over approximately one-quarter of the sky.

The dwarf galaxy population is highlighted in Figure 1, and includes several new members found as part of this observational campaign ([3,5-7, 11]). Figure 2 shows an updated version of Figure 5 from [8]. Here, we see that the majority of MW satellites have scale-radii close to around 100-200 kpc (with Fornax, Sextans and Canes Venatici being notable exceptions). M31 satellites have scale-radii as small as the MW population, and a large number with radii around $200-400 \mathrm{kpc}$. At brighter magnitudes

${ }^{a}$ e-mail: alan.mcconnachie@nrc-cnrc.gc.ca

This is an Open Access article distributed under the terms of the Creative Commons Attribution-Noncommercial License 3.0, which permits unrestricted use, distribution, and reproduction in any noncommercial medium, provided the original work is properly cited. 


\section{EPJ Web of Conferences}

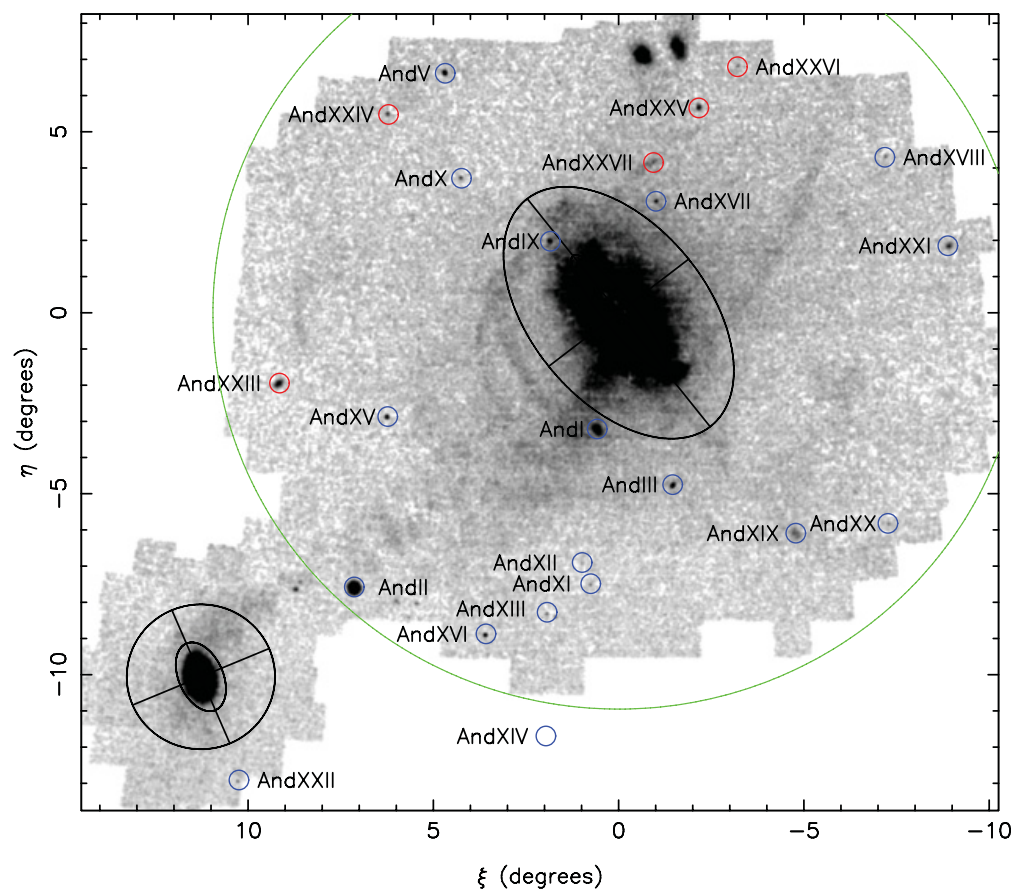

Figure 1. Spatial density distribution of candidate red giant branch stars in the environs of M31 within the PAndAS footprint. See [11].

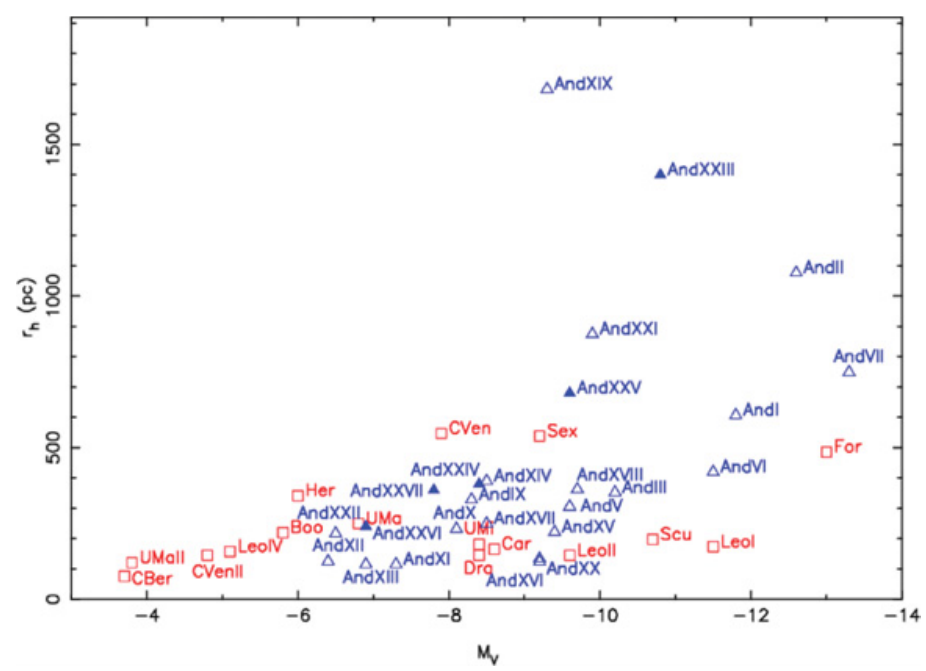

Figure 2. Updated version of Figure 5 from [8], showing the differences in scale size between the M31 satellite galaxies and those for the MW.

$\left(M_{V}<-8.5\right)$, the M31 system also has several dwarf galaxies with large scale-radii not seen around the MW $\left(600<r_{h}<1800 \mathrm{kpc}\right)$. Thus the overall sense is that the M31 population of dwarf galaxies are physically more extended in the mean than the MW population, which will likely also relate to their 


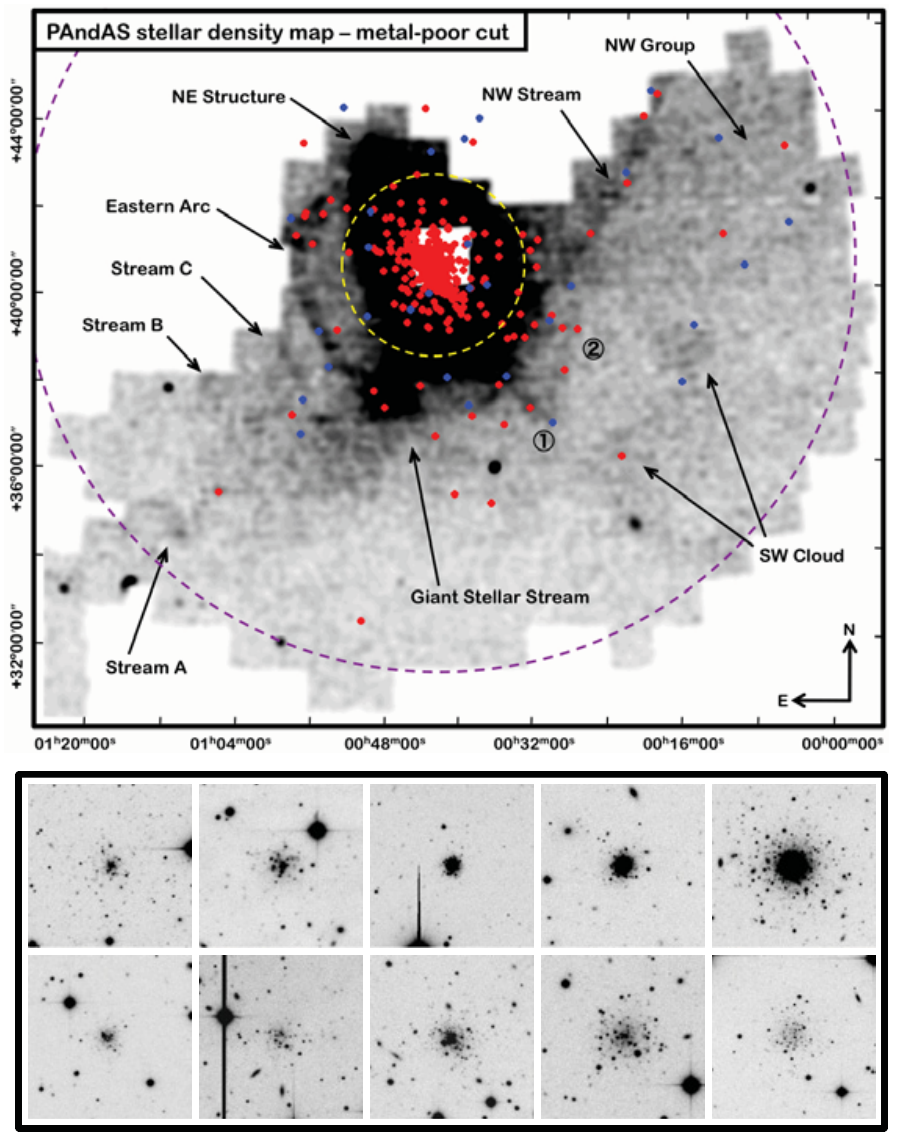

Figure 3. Spatial density distribution of globular clusters (red and blue dots for normal and extended clusters, respectively) compared to candidate red giant branch stars (for the dataset at the end of S09B). Examples of the globular clusters are shown in the lower panels and display a range of sizes and luminosities. See [4] (and also [2]).

kinematics (e.g., [1]) and underlying dark matter properties (e.g., [10]). The extent to which selection effects modify these conclusions has yet to be robustly demonstrated, but is work in progress.

\section{2 ...the globular cluster system}

Figure 3 is a reproduction of Figure 1 from [4], showing the spatial distribution of globular clusters (dots) compared to the stellar distribution (grey-scale). Red dots signify "normal" globular clusters, whereas blue dots signify the "extended" globular clusters around M31 first noticed by [2]. Lower panels show examples of the globular clusters in the dataset, and display a wide variety of sizes and luminosities.There is a clear correlation between the location of stellar substructures and the location of the outer $\left(R_{p}>50 \mathrm{kpc}\right)$ globular clusters, implying that the globular clusters may well have been brought into the M31 environment as satellites of the dwarf galaxies that were subsequently tidally disrupted and formed the stellar halo and visible substructures. This is similar to the idea first proposed by [12]. Also notable is the number of globular clusters in the outer regions of M31: only 9 globular clusters are known around the MW at radii greater than $38 \mathrm{kpc}$, and only two are known to lie at radii greater than $100 \mathrm{kpc}$. Clearly, the M31 outer cluster population is far richer in number and extent than for the MW. 
EPJ Web of Conferences

\section{References}

[1] Collins, M. L. M. 2011, Ph.D. Thesis

[2] Huxor, A. P., Tanvir, N. R., Irwin, M. J., Ibata, R., Collett, J. L., Ferguson, A. M. N., Bridges, T., \& Lewis, G. F. MNRAS 360, (2005) 1007

[3] Ibata, R., Martin, N. F., Irwin, M., Chapman, S., Ferguson, A. M. N., Lewis, G. F., \& McConnachie, A. W., ApJ, 671, (2007) 1591

[4] Mackey, A. D., Huxor, A. P., Ferguson, A. M. N., Irwin, M. J., Tanvir, N. R., McConnachie, A. W., Ibata, R. A., Chapman, S. C., \& Lewis, G. F., ApJL, 717, (2010) 11

[5] Martin, N. F., Ibata, R. A., Irwin, M. J., Chapman, S., Lewis, G. F., Ferguson, A. M. N., Tanvir, N., \& McConnachie, A. W., MNRAS, 371, (2006) 1983

[6] Martin, N. F., McConnachie, A. W., Irwin, M., Widrow, L. M., Ferguson, A. M. N., Ibata, R. A., Dubinski, J., Babul, A., Chapman, S., Fardal, M., Lewis, G. F., Navarro, J., \& Rich, R. M., ApJ, 705, (2009) 758

[7] McConnachie, A. W., Huxor, A., Martin, N. F., Irwin, M. J., Chapman, S. C., Fahlman, G., Ferguson, A. M. N., Ibata, R. A., Lewis, G. F., Richer, H., \& Tanvir, N. R., ApJ, 688, (2008) 1009

[8] McConnachie, A. W. \& Irwin, M. J. MNRAS, 365, (2006) 1263

[9] McConnachie, A. W., Irwin, M. J., Ibata, R. A., Dubinski, J., Widrow, L. M., Martin, N. F., Côté, P., Dotter, A. L., Navarro, J. F., Ferguson, A. M. N., Puzia, T. H., Lewis, G. F., Babul, A., Barmby, P., Bienaymé, O., Chapman, S. C., Cockcroft, R., Collins, M. L. M., Fardal, M. A., Harris, W. E., Huxor, A., Mackey, A. D., Peñarrubia, J., Rich, R. M., Richer, H. B., Siebert, A., Tanvir, N., Valls-Gabaud, D., \& Venn, K. A., Nature, 461, (2009) 66

[10] Pe narrubia, J., McConnachie, A. W., \& Navarro, J. F., ApJ, 672, (2008) 904

[11] Richardson, J. C., Irwin, M. J., McConnachie, A. W., Martin, N. F., Dotter, A. L., Ferguson, A. M. N., Ibata, R. A., Chapman, S. C., Lewis, G. F., Tanvir, N. R., \& Rich, R. M., ApJ, 732, (2011) 76

[12] Searle, L. \& Zinn, R., ApJ, 225, (1978) 357 